\title{
Designing robust rapid transit networks with alternative routes
}

\author{
Gilbert Laporte ${ }^{1}$, Angel Marín ${ }^{2}$, Juan A. Mesa ${ }^{3 *}$ and Federico Perea ${ }^{4}$ \\ ${ }^{1}$ Canada Research Chair in Distribution Management, HEC Montréal, Canada \\ ${ }^{2}$ Departamento de Matemática Aplicada y Estadística, Universidad Politécnica de Madrid, Spain \\ ${ }^{3}$ Departamento de Matemática Aplicada II, Universidad de Sevilla, Spain \\ ${ }^{4}$ Departamento de Métodos Estadísticos, Universidad de Zaragoza, Spain
}

\begin{abstract}
SUMMARY
The aim of this paper is to propose a model for the design of a robust rapid transit network. In this paper, a network is said to be robust when the effect of disruption on total trip coverage is minimized. The proposed model is constrained by three different kinds of flow conditions. These constraints will yield a network that provides several alternative routes for given origin-destination pairs, therefore increasing robustness. The paper includes computational experiments which show how the introduction of robustness influences network design. Copyright (C) 2010 John Wiley \& Sons, Ltd.
\end{abstract}

KEY WORDS: rapid transit systems; network design; robustness

\section{INTRODUCTION}

The increased demand for passenger transportation in and around urban areas and the resulting traffic congestion have lead many cities to build rapid transit systems and new conventional railway lines (see, e.g., [1]). Because of the high construction and operating costs of such systems, it is important to pay attention to issues affecting effectiveness and robustness at the planning stage. A crucial part of the planning process is the underlying infrastructure network design which consists of two intertwined problems: the determination of alignments and the location of stops and stations. Because most rapid transit systems are railways, we will use both terms indiscriminately.

Design decisions are considered at the strategic level, but they must incorporate the traffic behavior of users. At the upper level the objective is to maximize demand coverage, subject to design and budget constraints. At the lower level the traffic demand decisions are incorporated in the transit network design alternatives, considering the traffic cost of private and public modes, based on the system supply (the network to be constructed) and on assumptions made about the modal traffic costs. The selection and comparison of these alternatives may be carried out by considering that users choose both a path and a travel mode.

Several studies have addressed railway or metro network design problems. The location of a single alignment has been dealt with in the papers by Bruno et al. [2,3] and Dufourd et al. [4]. In the first one the weighted travel cost of all the users is minimized, while in the other two the coverage of the population by public network is maximized. The work of Laporte et al. [5], incorporates origindestination data in order to maximize the trip coverage. The papers of Hamacher et al. [6] and Laporte et al. [7] consider the problem of locating stations on a given alignment in different set-ups. The first one assumes that there already exists a partial alignment on which some stations are located, while the second one considers a discrete set of candidates to locate the stations. García and Marín [8,9] study the mode interchange and parking network design problems using bilevel programing. They address the multimodal traffic assignment problem with combined modes at a lower level. Laporte et al. [10]

*Correspondence to: Juan A. Mesa, Departamento de Matemática Aplicada II, Universidad de Sevilla, Spain.

E-mail: jmesa@us.es

Copyright (C) 2010 John Wiley \& Sons, Ltd. 
extend the previous models by incorporating station location and multiple alignments. Their model maximizes demand coverage, subject to budget constraints. Marín [11] studies the inclusion of a free but bounded number of lines, and each origin-destination (O/D) of the lines is chosen in the rapid transit network design model.

In a related field, a number of authors (see, e.g., Fortz and Labbé [12], Fortz et al. [13], and Grötschel et al. [14]) have studied the problem of designing robust low cost communication networks that can survive the failure of some edges. However, telecommunication and transportation networks operate differently. In telecommunication networks the routing of signals is decided by the network managers whereas in transportation networks passengers make their own choices. As a result the same solution methodology cannot apply to both types of problems.

Online planning in railway and other transportation networks needs to react in the best possible way to perturbations, and network robustness must be taken into account at the planning stage. Several sources of uncertainty are present in transportation systems. Stochasticity of demand has been addressed by many authors, e.g., Lou et al. [15] and Yin and Lawphongpanich [16]. We are concerned here with uncertainty relative to the network itself (see, e.g., [17]). The review article by Yang and Bell [18] considers link additions and link improvements in network design problems and casts these problems in the general framework of bilevel programing.

In the first case robust optimization is considered from a stochastic point of view by Rockafellar and Wets [19] who identify robust decision by discovering similarities among the optimal solutions for different scenarios. A framework for robust optimization is analyzed in Malcolm and Zenios [20] and Mulvey et al. [21]. These papers assess different choices for parameters while penalizing variance of the cost of deviations from feasibility. A serious difficulty in this approach is to strike a proper balance between the terms of the objective function. One must achieve a tradeoff between the mean and variance of the solution, and deviations from feasibility under all scenarios.

In the second case one seeks a solution that remains feasible even for worst-case scenario parameters. A bibliography on this topic, with comments on robustness in combinatorial optimization, can be found in Nikulin [22]. This optimization approach considers the robust counterpart of the problem, see for example, Ben-Tal and Nemirovski [23,24] in which a robust solution is sought using ellipsoidal uncertainty sets for data. The over-conservatism of this strategy is unsuitable for many situations in which only a low number of parameters are uncertain simultaneously $[25,26]$. This is in fact the case of railway network design problems in which robustness is considered according to disruptions to the usual operations [27,28]. In general, the robustness of a system indicates the influence of the perturbations on the usual functioning. The more a system is capable of achieving its aim in adverse conditions, the more robust it becomes. The perturbation on the operations of a railway system can come from internal causes (signals or rolling stock failures, crew problems, coordination and computer problems, etc.) or from external causes (extreme weather conditions, a drop in electrical power, etc.). The occurrence of two or more perturbations at the same time is very infrequent, but since lines use several sections of the network, a disruption on one arc affects the operation on others, causing secondary or knock-on delays.

Our goal is to design robust transportation networks. We consider that a railway network is robust when, in the event of arc failures, a high proportion of the passengers will still find the network useful and faster than other means of transportation. Therefore, we will build networks that provide several routes to passengers, thus increasing robustness. Although flow variables are introduced in our models, the reader should note that such variables are used only for modeling purposes, and to define the different routes available to each O/D pair on the RTN, but users always have the final say when it comes to deciding which route to take, in accordance with the first Wardrop principle. In principle, modeling user behavior can be done through the use of variational inequalities or of mathematical programs with a rather large number of equality constraints (see, e.g., [29]). But given the very high computational complexity of these models, we have avoided the use of bilevel programing in the RTND model. This choice is coherent with the assumption that the system capacity is not considered in our model. Nevertheless, our designs will provide users with several possibilities. Some models can be used to design networks that maximize trip coverage under normal conditions, i.e., no disruptions occur. Others maximize trip coverage in the presence of disruptions. We propose a combination of these two objectives, with the aim of providing a near-optimal network both when no failures occur and when an arc is inoperative. 
The remainder of this paper is organized as follows. Section 2 presents the model for the core network design problem. Section 3 is dedicated to the inclusion in the model of the proposed robustness constraints. Section 4 describes computational experiments. The paper ends with some conclusions.

\section{A MODEL FOR THE RAPID TRANSIT NETWORK DESIGN PROBLEM}

In our model for the Rapid Transit Network Design Problem (RTNDP) we assume that the mobility patterns in a metropolitan area are known. This implies that the number of potential passengers from each origin to each destination is given. We also assume that the locations of the potential stations are given. There already exists a different mode of transportation (for example, private cars) competing with the railway. When deciding which mode each demand is allocated to, the comparison between the generalized costs of the travelers is used. The aim of the model is to design a network, i.e., to decide at which nodes to locate the stations and how to connect them, consisting of railway lines, and covering as many trips as possible. Since resources are limited we also impose a budget constraint on construction costs.

Similar ILP models have appeared in the literature (see [10,30]). In these models, flow variables on the routes through the RTN are binary, therefore allowing only to decide whether an O/D pair is assigned to the RTN or not. In our model we allow these variables to take any value in $[0,1]$ and add flow variables on the alternative mode. This way we allow that part of the demand of the O/D pair is routed through the RTN and the rest through the alternative mode, thus allowing our model to become robust when providing O/D pairs with different alternative routes in the RTN (see Section 3).

\subsection{Data and notation}

The model uses the following notation:

(1) A set $N=\left\{n_{i}: i=1,2 \ldots, n\right\}$ of potential sites for locating stations.

(2) A set $A^{\prime}$ of feasible (bidirectional) arcs linking the elements in $N$. Therefore, we have a graph $G^{\prime}=\left(N, A^{\prime}\right)$, from which arcs are to be selected to form railway lines. Furthermore, there exists a graph $G^{\prime \prime}=\left(N, A^{\prime \prime}\right)$ representing the network used by the complementary mode (e.g., the street network). Let $G=(N, A)$, where $A=A^{\prime} \cup A^{\prime \prime}$, be the whole network. Denote by $N(i)=\left\{n_{j}: \exists a \in A^{\prime}, a=\left(n_{i}, n_{j}\right)\right\}$ the set of nodes adjacent to $n_{i}$.

(3) Every feasible arc $a=\left(n_{i}, n_{j}\right) \in A^{\prime}$ has an associated length $d_{i j}$ equal to approximate Euclidean distances if the system to be designed is underground, and to street network distances if it is at grade. However, forbidden regions will increase distances, and $d_{i j}$ can also be interpreted as the generalized cost (time) of traversing arc $a=\left(n_{i}, n_{j}\right) \in A^{\prime}$.

(4) For every node $n_{i}$ and every arc $a \in A^{\prime}$ there is an associated cost of constructing the corresponding infrastructure: $c_{i}$ is the cost of building a station at node $n_{i}$, and $c_{a}$ is the cost of building link $a$. A bound $C_{\max }$ on the available budget is also given.

(5) The demand pattern is given by a vector $\left(g_{w}\right): w \in W$, where $W$ is the set of ordered O/D pairs: $W=\left\{w=(p, q): n_{p}, n_{q} \in N\right\}$.

(6) The generalized cost of satisfying every demand by the railway network and the complementary modes are $u_{w}^{\mathrm{RN}}$ and $u_{w}^{\mathrm{COM}}$, respectively. While the cost of using the complementary mode depends on its network and therefore is an input data, the cost of using the railway depends on the topology of the network to be constructed. The computation of railway costs $u_{w}^{\mathrm{RN}}$ can be done by adding the lengths of the arcs of the path of $w$ in the railway network. Let $u_{w}$ be the generalized cost of $w$ either by $G^{\prime}$ or by $G^{\prime \prime}$.

The aim of the model is to design a network consisting of a set $L$ of lines, $|L|$ being a low number. Since constraints on the total cost will be imposed, we will allow some lines of $L$ to not be included in the designed network. Note that for the model to be meaningful we need to impose technical constraints on the data: both the lengths $d_{i j}$ on $A^{\prime}$ and the complementary mode $\operatorname{cost} u_{w}^{\mathrm{COM}}$ must satisfy the triangle inequality. 


\subsection{Variables}

The following variables will be used in the model:

- $y_{i}^{l}=1$, if node $n_{i}$ is a station of line $l ; 0$ otherwise.

- $x_{i j}^{l}=1$, if the arc $a=\left(n_{i}, n_{j}\right) \in A^{\prime}$ belongs to line $l \in L ; 0$ otherwise.

- $x_{\mathrm{ij}}$, if the arc $a=\left(n_{i}, n_{j}\right) \in A^{\prime}$ belongs to the railway network; 0 otherwise.

- $\tilde{f}_{i j}^{w}$ denotes the proportion the demand of $w \in W$ going through $\operatorname{arc}\left(n_{i}, n_{j}\right) \in A^{\prime}$, from $n_{\mathrm{i}}$ to $n_{j}$, $f_{i j}^{w} \in[0,1]$, if no failure occurs. Note that these variables will define the fastest route for $w$ in the network to be built.

- $\tilde{\varphi}_{i j}^{w}$ denotes the proportion of the demand of $w \in W$ through $\operatorname{arc}\left(n_{i}, n_{j}\right) \in A^{\prime}$, from $n_{i}$ to $n_{j}, \tilde{\varphi}_{i j}^{w} \in[0,1]$, if no failure occurs.

- $h_{l}=1$, if line $l$ is included; 0 otherwise.

- $p_{w}=1$, if the demand of $w$ is allocated to the railway network, that is, if its fastest route in the network takes less time than that of the alternative mode; 0 , otherwise.

In practice, variables $\tilde{f}_{i j}^{w}$ and $\tilde{\varphi}_{i j}^{w}$ often take integer values, unless more than one route take equal time.

\subsection{Objective function and constraints}

The objective of our model is to maximize the railway demand coverage when no disruption occurs:

$z_{1}=\sum_{w=(p, q) \in W} g_{w} p_{w}$

The constraints are as follows:

- Budget constraints:

$$
\sum_{\left(n_{i}, n_{j}\right) \in A^{\prime}, i<j} c_{i j} x_{i j}+\sum_{l \in L} \sum_{\eta_{i} \in N} c_{i} y_{i}^{l} \leq C_{\max } .
$$

- Alignment location constraints:

$$
\begin{aligned}
& x_{i j}^{l} \leq y_{i}^{l},\left(n_{i}, n_{j}\right) \in A^{\prime}, i<j, l \in L \\
& x_{i j}^{l} \leq y_{j}^{l},\left(n_{i}, n_{j}\right) \in A^{\prime}, i<j, l \in L \\
& x_{i j}^{l}=y_{j i}^{l},\left(n_{i}, n_{j}\right) \in A^{\prime}, i<j, l \in L \\
& x_{i j}^{l} \leq x_{i j}^{l},\left(n_{i}, n_{j}\right) \in A^{\prime}, i<j, l \in L \\
& x_{i j}^{l} \leq \sum_{l \in L} x_{i j}^{l},\left(n_{i}, n_{j}\right) \in A^{\prime}, i<j \\
& \sum_{j \in N(i)} x_{i j}^{l} \leq 2, n_{i} \in N, l \in L \\
& h_{l}+\sum_{\left(n_{i}, n_{j}\right) \in A^{\prime}, i<j} x_{i j}^{l}=\sum_{n_{i} \in N} y_{i}^{l}, l \in L \\
& \frac{1}{2}-\sum_{\left(n_{i}, n_{j}\right) \in A^{\prime}, i<j} x_{i j}^{l}+M\left(h_{l}-1\right) \leq 0, l \in L \\
& \frac{1}{2}-\sum_{\left(n_{i}, n_{j}\right) \in A^{\prime}, i<j} x_{i j}^{l}+M h_{l} \geq 0, l \in L \\
& \sum_{n_{i} \in B} \sum_{n_{j} \in B} x_{i j}^{l} \leq|B|-1, B \subseteq N,|B| \geq 2, l \in L .
\end{aligned}
$$


Routing demand conservation constraints:

$$
\begin{aligned}
& \sum_{\left(n_{i}, n_{p}\right) \in A^{\prime}} \tilde{f}_{i p}^{w}+\sum_{\left(n_{i}, n_{p}\right) \in A^{\prime \prime}} \tilde{\varphi}_{i p}^{w}=0, w=(p, q) \in W \\
& \sum_{\left(n_{p}, n_{j}\right) \in A^{\prime}} \tilde{f}_{p j}^{w}+\sum_{\left(n_{p}, n_{j}\right) \in A^{\prime \prime}} \tilde{\varphi}_{p j}^{w}=1, w=(p, q) \in W \\
& \sum_{\left(n_{i}, n_{q}\right) \in A^{\prime}} \tilde{f}_{i q}^{w}+\sum_{\left(n_{i}, n_{q}\right) \in A^{\prime \prime}} \tilde{\varphi}_{i q}^{w}=1, w=(p, q) \in W \\
& \sum_{\left(n_{q}, n_{j}\right) \in A^{\prime}} \tilde{f}_{q j}^{w}+\sum_{\left(n_{q}, n_{j}\right) \in A^{\prime \prime}} \tilde{\varphi}_{q j}^{w}=0, w=(p, q) \in W \\
& \sum_{\left(n_{i}, n_{k}\right) \in A^{\prime}} \tilde{f}_{i k}^{w}-\sum_{\left(n_{k}, n_{j}\right) \in A^{\prime \prime}} \tilde{f}_{k j}^{w}=0, \text { if } k \notin\{p, q\}, w=(p, q) \in W \\
& \sum_{\left(n_{i}, n_{k}\right) \in A^{\prime \prime}} \tilde{\varphi}_{i k}^{w}-\sum_{\left(n_{k}, n_{j}\right) \in A^{\prime \prime}} \tilde{\varphi}_{k j}^{w}=0, \text { if } k \notin\{p, q\}, w=(p, q) \in W \\
& \tilde{f}_{i j}^{w}+\tilde{\varphi}_{q j}^{w} \leq 1,\left(n_{i}, n_{j}\right) \in A, w \in W .
\end{aligned}
$$

- Location-allocation constraints:

$$
\tilde{f}_{i j}^{w}+p_{w}-1 \leq \sum_{l \in L} x_{i j}^{l},\left(n_{i}, n_{j}\right) \in A^{\prime}, w \in W .
$$

- Splitting demand constraints:

$$
\varepsilon+u_{w}-u_{w}^{\mathrm{COM}}-M\left(1-p_{w}\right) \leq 0, w=(p, q) \in W .
$$

where $u_{w}=\sum_{\left(n_{i}, n_{j}\right) \in A^{\prime}} d_{i j} \tilde{f}_{i j}^{w}+\sum_{\left(n_{i}, n_{j}\right) \in A^{\prime \prime}} u_{i j}^{\mathrm{COM}} \tilde{\varphi}_{i j}^{w}, M$ is a large real number, $\varepsilon>0$ is a small tolerance, and $u_{i j}^{\mathrm{COM}}$ is the generalized cost of traversing arc $\left(n_{i}, n_{j}\right)$ by the complementary mode.

Constraint (1) is the budget constraint. Constraints (2) and (3) ensure that a link is included in the railway network only if the nodes to which the link is incident are also selected. Constraints (4) allow edges to be used in both directions. Constraints (5) and (6) state that arc $\left(n_{i}, n_{j}\right)$ is built if and only if a line uses it. Constraints (7) force each node to have at most two associated edges of each line. Constraints (11) ensure that a line does not contain a cyclic subgraph. Note that these constraints along with (8) guarantee that the lines to be constructed will induce paths. However, a line of the network must have at least one edge, which is ensured by constraints (9). If a line $l$ is not considered in the design then it does not have any edge by (10). Constraints (12)-(18) are the flow conservation constraints at each node. The incoming flow equals the outgoing flow for both pairs of variables. We also impose that both outgoing flows and both incoming flows must be equal to 1 at the beginning and at the end of the paths, respectively. Constraints (19) guarantee that an O/D pair demand is routed on an edge only if this edge belongs to the railway network. Finally, constraints (20) force the demand to be assigned to the railway mode if and only if the associated cost of using this network (taking the fastest route) does not exceed the corresponding cost of the complementary mode. The role of $\varepsilon$ in these constraints is to break ties. For the sake of simplicity mode choice was reduced to a $0-1$ decision. However, our model admits a more elaborated mode distribution as that applied in the paper by Marín and García-Ródenas [31] where a piecewise linear approximation of a logit function is considered. For computational reasons, transfer costs are not taken into account in this model since the model is already rather heavy. A model in which transfers are considered is provided in Garzón et al. [32].

\section{INTRODUCING ROBUSTNESS IN THE MODEL}

Our model for the design of a railway network does not consider the system capacity. This is consistent with the assumption that public transportation costs are independent of link passenger flows, for which we assume that the available capacity is sufficient. This context is one for which 
the user-link assignments are all-or-nothing. This leads to concentrating riders for each O/D pair in single routes. Since this type of solution usually implies that some parts of the network are crowded while others are almost empty, the RTNDP model has no robust network solution and any disruption of service, especially in the most crowded sections of the network, involves a large number of passengers. The introduction of the robustness constraints not only provides alternative routes in the event of arc failure, but it also helps avoid congestion on a restricted set of arcs.

To incorporate the robustness constraints in the model, we introduce the following variables:

- $f_{i j}^{w}$ denotes the proportion of the demand of $w \in W$ that goes through $\operatorname{arc}\left(n_{i}, n_{j}\right) \in A^{\prime}$, from $n_{i}$ to $n_{j}$, $f_{i j}^{w} \in[0,1]$, if a failure occurs.

- $\varphi_{i j}^{w}$ denotes the proportion of the demand of $w \in W$ that travels through arc $\left(n_{i}, n_{j}\right) \in A^{\prime \prime}$, from $n_{i}$ to $n_{j}$, $\varphi_{i j}^{w} \in[0,1]$, if a failure occurs.

These variables are created in order to provide O/D pairs with different alternative routes. They just seem a duplication of the $\tilde{f}, \tilde{\varphi}$ variables, but they are in fact crucial to our definition of robustness. Although the new variables are defined as percentage of the demand of each O/D pair going through certain arcs, they should be seen as alternative routes, since users have the power to decide which route to take.

The objective now consists not only of maximizing demand coverage, but it also takes into account the effects of possible failures in the network. Therefore, apart from $z_{1}$, two other customer-oriented criteria are combined in the objective function:

- The maximization of the railway demand coverage in case of failures:

$$
z_{2}=\sum_{w=(p, q) \in W} g_{w}\left(\sum_{j \in N(p),\left(n_{p}, n_{j}\right) \in A^{\prime}} f_{p j}^{w}\right) .
$$

Note that $\sum_{j \in N(p),\left(n_{p}, n_{j}\right) \in A^{\prime}} f_{p j}^{w}$ is the proportion of the demand of the O/D pair $w=(p, q)$ that will use the railway network.

- The minimization of the total routing maximal value:

$$
z_{\text {DIST }}=\sum_{w \in W,(i, j) \in A^{\prime}} f_{i j}^{w} d_{i j}
$$

The objective function becomes

$z=\alpha z_{1}+(1-\alpha) z_{2}-\beta z_{\text {DIST }}$.

where $\beta$ is a positive number close to 0 , to keep $z_{1}$ and $z_{2}$ as primary goals. If $\alpha \in[0,1]$ is close to 1 , the objective is to maximize the demand coverage when no arc fails. In contrast, if $\alpha$ is close to 0 , the objective is to maximize the demand coverage when arcs fail. We add the term $z_{\mathrm{DIST}}$ to force the model to choose the shortest of all potential routes (still keeping as primary goal the demand coverage). The model has the same structure as that of Section 2.3, with the following changes. Constraints (12)-(18) become

- Routing demand conservation constraints:

$$
\begin{aligned}
& \sum_{\left(n_{i}, n_{p}\right) \in A^{\prime}} f_{i p}^{w}+\sum_{\left(n_{i}, n_{p}\right) \in A^{\prime \prime}} \varphi_{i p}^{w}=0, w=(p, q) \in W \\
& \sum_{\left(n_{p}, n_{j}\right) \in A^{\prime}} f_{p j}^{w}+\sum_{\left(n_{p}, n_{j}\right) \in A^{\prime \prime}} \varphi_{p j}^{w}=1, w=(p, q) \in W \\
& \sum_{\left(n_{i}, n_{q}\right) \in A^{\prime}} f_{i q}^{w}+\sum_{\left(n_{i}, n_{q}\right) \in A^{\prime \prime}} \varphi_{i q}^{w}=1, w=(p, q) \in W
\end{aligned}
$$




$$
\begin{aligned}
& \sum_{\left(n_{q}, n_{j}\right) \in A^{\prime}} f_{q i}^{w}+\sum_{\left(n_{q}, n_{j}\right) \in A^{\prime \prime}} \varphi_{q i}^{w}=0, w=(p, q) \in W \\
& \sum_{\left(n_{i}, n_{k}\right) \in A^{\prime}} f_{i k}^{w}-\sum_{\left(n_{k}, n_{j}\right) \in A^{\prime}} f_{k j}^{w}=0, \text { if } k \notin\{p, q\}, w=(p, q) \in W \\
& \sum_{\left(n_{i}, n_{k}\right) \in A^{\prime \prime}} \varphi_{i k}^{w}-\sum_{\left(n_{k}, n_{j}\right) \in A^{\prime \prime}} \varphi_{k j}^{w}=0, \text { if } k \notin\{p, q\}, w=(p, q) \in W \\
& f_{i j}^{w}+\varphi_{i j}^{w} \leq 1,\left(n_{i}, n_{j}\right) \in A, w \in W \\
& M f_{i j}^{w} \geq \tilde{f}_{i j}^{w},\left(n_{i}, n_{j}\right) \in A, w \in W .
\end{aligned}
$$

Constraints (21)-(26) are flow conservation constraints (as for variables $\tilde{f}$ and $\tilde{\varphi}$ ). Constraints (28) state that the fastest route for an O/D pair (defined by variables $\tilde{f}$ and $\tilde{\varphi}$ ) must be among the different alternative routes chosen for that pair. Constraints (19) become:

$f_{i j}^{w}+p_{w}-1 \leq \sum_{l \in L} x_{i j}^{l},\left(n_{i}, n_{j}\right) \in A^{\prime}, w \in W$.

since we must now build the arcs for every possible route.

\subsection{Demand-arc flow constraints}

The first kind of robustness constraints to be introduced are called demand-arc flow constraints (DAF) and allow the demand of every arc to be routed through alternative paths, considering the complementary mode as an alternative. A disadvantage of multi-path routing lies in the fact that the demand is split, and therefore a more expensive but more robust solution arises. The user cost difference between the standard RTNDP and the RTNDP with demand-arc constraints is the price of robustness [26].

These constraints, called demand-arc flow constraints, DAF for short, allow the demand of every arc of every O/D pair $w$ to use at least $r_{i j}^{w}$ different routes. Therefore,

$f_{i j}^{w} \leq \frac{1}{r_{i j}^{w}},\left(n_{i}, n_{j}\right) \in A_{\mathrm{DAF}}^{\prime} \subseteq A^{\prime}, w \in W_{\mathrm{DAF}} \subseteq W$.

where $A_{\mathrm{DAF}}^{\prime}$ and $W_{\mathrm{DAF}}$ are the sets of selected arcs and O/D pairs, respectively. The limitation can be uniform so that $r_{i j}^{w}=r, \forall\left(n_{i}, n_{j}\right) \in A_{\mathrm{DAF}}^{\prime}, w \in W_{\mathrm{DAF}}$. If $r$ is a positive integer, then these constraints impose that each affected O/D pair will have $r$ different alternative routes in the network (if this is allowed by the budget constraint).

To illustrate, consider the example of Figure 1 where demand $(1,3)$ takes the direct route $f_{1,3}^{1,3}=1$. If we impose DAF constraints with $r=2$, this demand now has two possible routes: directly from $n_{1}$ to $n_{3}$, or via node 2 as depicted in Figure 2, that is, $f_{1,3}^{1,3}=f_{1,2}^{1,3}=f_{2,3}^{1,3}=0.5$.

\subsection{Arc flow constraints}

An interpretation of the arc flow constraints, AF for short, is that only a percentage of the total number of O/D pairs will have their demand routed through the arcs involved. In case of arc failure, only a percentage of the O/D flows will be affected

$$
\sum_{w \in W_{\mathrm{AF}}} f_{i j}^{w}+\sum_{w \in W_{\mathrm{AF}}} f_{j i}^{w} \leq \frac{\left|W_{\mathrm{AF}}\right|}{r_{i j}},\left(n_{i}, n_{j}\right) \in A_{\mathrm{AF}}^{\prime} \subseteq A^{\prime}, W_{\mathrm{AF}} \subseteq W .
$$

where $r_{i j}$ plays the same role as $r_{i j}^{w}$ and $A_{\mathrm{AF}}^{\prime}$ and $W_{\mathrm{AF}}$ are the subset of arcs and the subset of O/D pair that will be considered, respectively. A common situation arises when the upper bound is uniform $r_{i j}=r, \forall\left(n_{i}, n_{j}\right) \in A_{\mathrm{AF}}^{\prime}$. Note that this constraint relaxes the constraint on demand-arc flow, and may only affect some arcs, for example, the hub transit network. Another possibility is to consider only a selected group of O/D pairs, for example, O/D pairs with a long distance between the origin and the destination, or pairs without private car availability. 


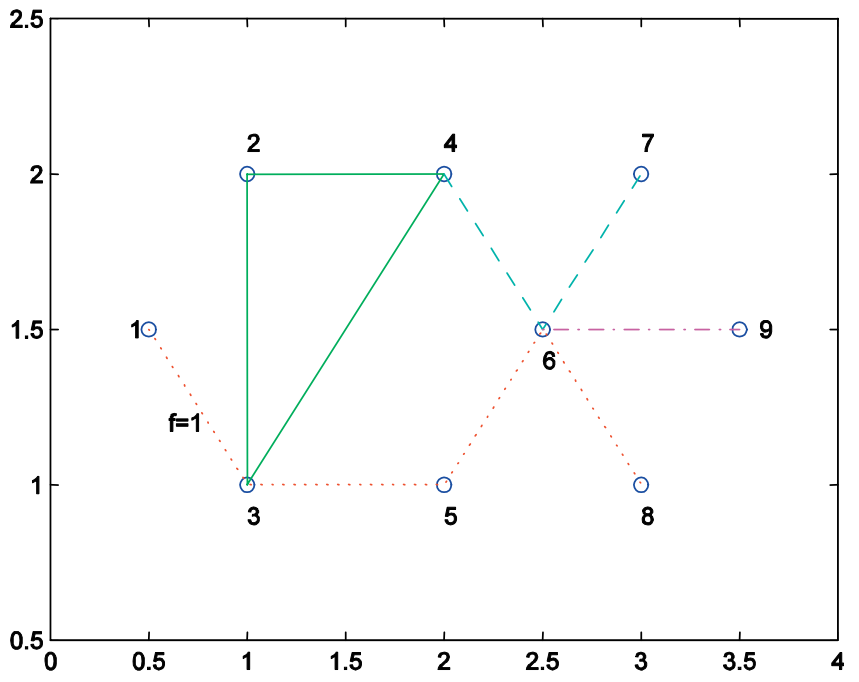

Figure 1. Lines of the network and flow from $n_{1}$ to $n_{3}$ when $r=1$ and $C_{\max }=40$.

\subsection{Arc demand constraints}

When imposing arc demand constraints, $\mathrm{AD}$ for short, only a percentage of the total traffic may be routed through the selected arcs. These constraints imply that the O/D demands are spread over a number of arcs, thus avoiding their concentration and railway congestion.

$$
\sum_{w \in W_{\mathrm{AD}}} f_{i j}^{w} g_{w}+\sum_{w \in W_{\mathrm{AD}}} f_{j i}^{w} g_{w} \leq \frac{\Sigma_{w \in W} g_{w}}{r_{i j}},\left(n_{i}, n_{j}\right) \in A_{\mathrm{AD}}^{\prime} \subseteq A^{\prime}, W_{\mathrm{AD}} \subseteq W .
$$

where $A^{\prime}{ }_{\mathrm{AD}}$ and $W_{\mathrm{AD}}$ are the subset of arcs and the subset of $\mathrm{O} / \mathrm{D}$ pairs to be considered, respectively. As before, it is often the case that $r_{i j}=r, \forall(i, j) \in A_{\mathrm{AD}}^{\prime}$. Also, as for the previous constraints and for the same reason, a selected group of O/D pairs to which the constraints are applied may be selected. It should be stressed that the flow variables $f$ and $\varphi$ are only introduced for computational purposes and to define the different routes on the RTN for each O/D pair, but users are free to choose their route (we assume they will choose the fastest one available). For instance, in Figure 2 the flow from node 1 to

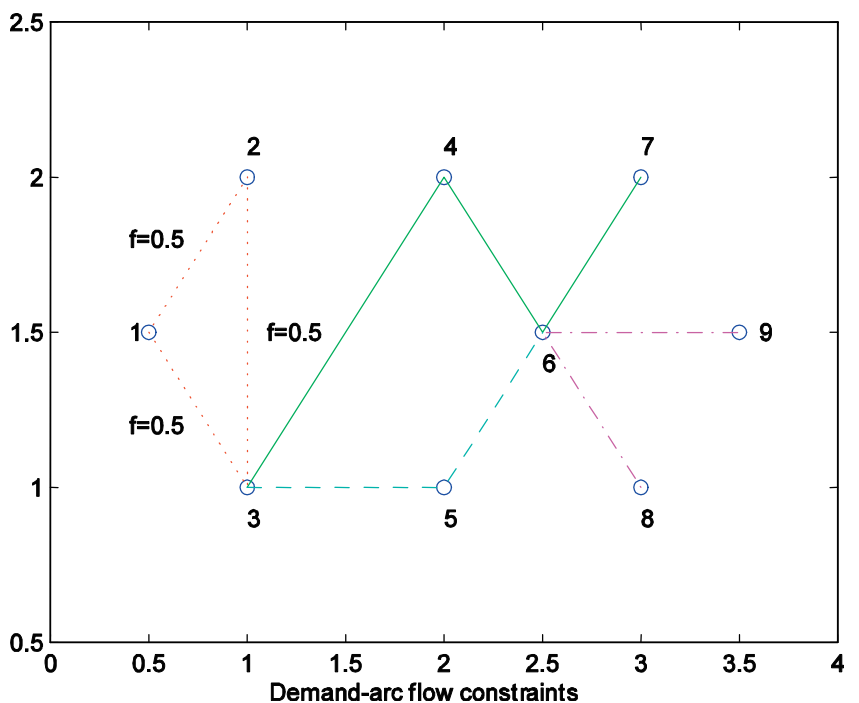

Figure 2. Lines of the network and flow from $n_{1}$ to $n_{3}$ when $r=2$ and $C_{\max }=40$. 
node 3 is split into two. This does not mean that half of the users go via one route and the other half via the other route, but only that users can choose either alternative, and, if one of them is not available, users can still find the RTN attractive.

\section{COMPUTATIONAL EXPERIMENTS}

Our computational experiments aim to assess to which extent the parameter $\alpha$ affects the objective function and the resulting network. The model was solved by branch-and-bound, which was implemented in GAMS 22.2 and CPLEX 10.0. The network depicted in Figure 3 was used for the experiments. Each node has an associated construction $\operatorname{cost} c_{i}$, and a pair $\left(c_{i j}, d_{i j}\right)$ is associated to each edge, where $c_{i j}$ and $d_{i j}$ are the construction cost of the edge and its length. The latter parameter can also represent the generalized cost of traversing the edge using the railway system.

The O/D demands $g_{w}$ and the cost $u_{w}^{\mathrm{COM}}$ for each O/D pair $w \in W$ are given by the following matrices:

$$
\begin{aligned}
& G=\left(\begin{array}{llllllllc}
0 & 9 & 26 & 19 & 13 & 12 & 6 & 6 & 4 \\
11 & 0 & 14 & 26 & 7 & 18 & 3 & 7 & 9 \\
30 & 19 & 0 & 30 & 24 & 8 & 3 & 9 & 11 \\
21 & 9 & 11 & 0 & 22 & 16 & 21 & 18 & 16 \\
14 & 14 & 8 & 9 & 0 & 20 & 12 & 18 & 9 \\
26 & 1 & 22 & 24 & 13 & 0 & 11 & 28 & 21 \\
7 & 5 & 6 & 19 & 15 & 13 & 0 & 16 & 14 \\
5 & 9 & 11 & 16 & 17 & 25 & 17 & 0 & 21 \\
6 & 8 & 10 & 18 & 11 & 20 & 14 & 20 & 0
\end{array}\right) ; \\
& u_{w}^{\text {COM }}= \\
&\left(\begin{array}{llllllllll}
0 & 1,6 & 0,8 & 2 & 1,6 & 2,5 & 4 & 3,6 & 4,6 \\
2 & 0 & 0,9 & 1,2 & 1,5 & 2,5 & 3,2 & 3,5 & 4,5 \\
1,5 & 1,4 & 0 & 1,3 & 0,9 & 2 & 3,3 & 2,9 & 3,9 \\
1,9 & 2 & 1,9 & 0 & 1,8 & 2 & 2 & 3,8 & 4,1 \\
3 & 1,5 & 2 & 2 & 0 & 1,5 & 3 & 2 & 3 \\
2,1 & 2,7 & 2,2 & 1 & 1,5 & 0 & 2,5 & 3 & 2,5 \\
3,9 & 3,9 & 3,9 & 2 & 3 & 2,5 & 0 & 2,5 & 2,5 \\
5 & 3,5 & 4 & 4 & 2 & 3 & 2,5 & 0 & 2,5 \\
4,6 & 4,5 & 4 & 3,5 & 3 & 2,5 & 2,5 & 2,5 & 0
\end{array}\right) ;
\end{aligned}
$$

As usual, subtour elimination constraints (11) where initially relaxed and gradually imposed, except in the case of circular lines.

Table I summarizes our results. We have used $C_{\max }=40$, and we have imposed the three different robustness constraints separately, over the central arcs, $(2,3),(2,4),(3,4),(3,5),(4,5)$. The resulting networks are: $R 1=\{(1,3,4,7,6,8),(3,5,6,9)\}$, and $R 2=\{(4,6,7),(6,8),(1,2,3,1),(3,5,6,9)\}$.

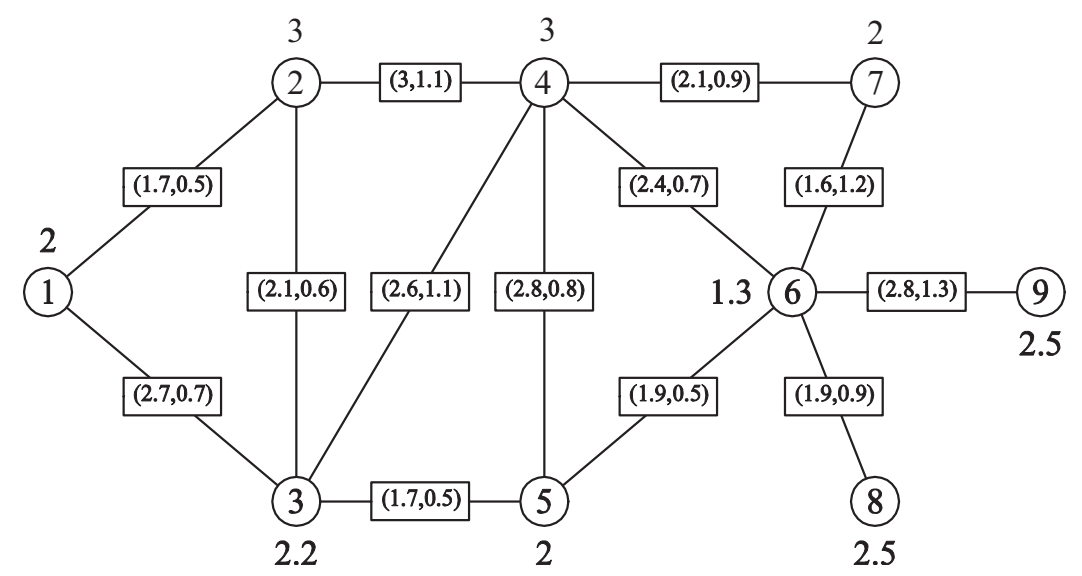

Figure 3. Test network. 
Table I. Traffic covered by the railway network when no failure occurs and when failures occur, and resulting networks imposing the three different robustness constraints for different values of $\alpha$.

\begin{tabular}{|c|c|c|c|c|c|c|c|c|c|}
\hline \multirow[t]{2}{*}{$\alpha$} & \multicolumn{3}{|c|}{ DAF, $r=2$} & \multicolumn{3}{|c|}{$\mathrm{AF}, r=10$} & \multicolumn{3}{|c|}{$\mathrm{AD}, r=10$} \\
\hline & $\mathrm{z}_{1}$ & $\mathrm{z}_{2}$ & $R$ & $\mathrm{z}_{1}$ & $\mathrm{z}_{2}$ & $R$ & $\mathrm{z}_{1}$ & $\mathrm{z}_{2}$ & $R$ \\
\hline 0 & 792 & 792 & $R 1$ & 792 & 716.2 & $R 1$ & 792 & 659.16 & $R 1$ \\
\hline 0.25 & 792 & 792 & $R 1$ & 792 & 716.2 & $R 1$ & 831 & 652.1 & $R 2$ \\
\hline 0.5 & 792 & 792 & $R 1$ & 831 & 683.02 & $R 2$ & 831 & 652.1 & $R 2$ \\
\hline 0.75 & 792 & 792 & $R 1$ & 831 & 683.02 & $R 2$ & 831 & 652.1 & $R 2$ \\
\hline 1 & 831 & 658.9 & $R 2$ & 831 & 683.02 & $R 2$ & 831 & 652.1 & $R 2$ \\
\hline
\end{tabular}

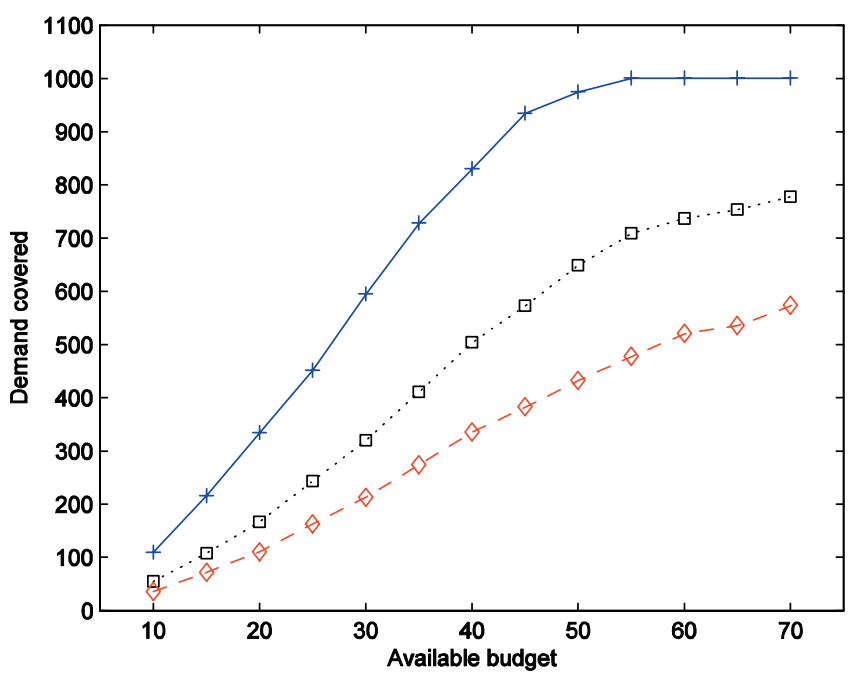

Figure 4. Demand-arc flow constraints for $r=1(+), r=2(\square), r=3(\diamond)$.

Figure 4 shows the asymptotic behavior of trip coverage with respect to budget when imposing DAF constraints for different values of $r$. One can see that in certain cases it is not worth investing more money in the network, since the increase in trip coverage is too low. Other observations are:

- The higher the value of $r$, the lower the covered traffic.

- The higher the value of $r$, the smoother the increase of the covered traffic. Thus, increasing robustness makes the network less profitable in terms of trip coverage.

- The maximum curvature decreases as the robustness of the network increases. The value of the budget which a larger starts triggering of trip coverage increases with $r$.

We are aware that our experiments are limited by the size of the instances that can be solved optimally. Our aim was not to solve large-scale instances, but rather to illustrate the feasibility of integrating robustness considerations in a planning model. Metaheuristics should likely be used for larger instances. Work along these lines is presented in Garzón et al. [33]. Another possibility is to use decomposition methods [30].

\section{CONCLUSIONS}

An integer linear programing model for building railway networks in competition with previously built networks was developed. Three ways of introducing robustness by capacity constraints were also studied. Experiments on robustness constraints were conducted on a small network. Further research avenues include improving the RTNDP model in different ways, namely by considering the multinomial logit mode demand distribution (see [31]) or constructing stochastic assignment model 
including the possibility of arc failure in the arc travel time stochasticity [34]. In addition, powerful metaheuristics could be developed to handle realistic size instances.

\section{ACKNOWLEDGEMENTS}

This work was partially supported by the Future and Emerging Technologies Unit of EC (IST priority -6th FP), under contract no. FP6-021235-2 (project ARRIVAL), by the Ministerio de Fomento (Spain) under project PT2007-003-08CCPP, by the Ministerio de Educación y Ciencia (Spain) under projects TRA2005-09068-C03-01 and MTM2006-15054, by the Ministerio de Ciencia e Innovación (Spain) under project TRA2008-06782-C02-01, and by the Canadian Natural Sciences and Engineering Research Council under grant 39682-05. This support is gratefully acknowledged. Thanks are due to two referees for their valuable comments.

\section{REFERENCES}

1. Gendreau M, Laporte G, Mesa JA. Locating rapid transit lines. Journal of Advanced Transportation 1995; 29:145162.

2. Bruno G, Ghiani G, Improta G. A multi-modal approach to the location of a rapid transit line. European Journal of Operational Research 1998; 104:321-332.

3. Bruno G, Gendreau M, Laporte G. A heuristic for the location of a rapid transit line. Computers \& Operations Research 2002; 29:1-12.

4. Dufourd H, Gendreau M, Laporte G. Locating a transit line using tabu search. Location Science 1996; 4:1-19.

5. Laporte G, Mesa JA, Ortega FA, Sevillano I. Maximizing trip coverage in the location of a single rapid transit alignment. Annals of Operations Research 2005; 136:49-63.

6. Hamacher H, Liebers A, Schöbel A, Wagner D, Wagner F. Locating new stops in a railway network. Electronic Notes in Theoretical Computer Science 2001; 50:11.

7. Laporte G, Mesa JA, Ortega FA. Locating stations on rapid transit lines. Computers \& Operations Research 2002; 29:741-759.

8. García R, Marín A. Urban multimodal interchange design methodology. In Mathematical Methods on Optimization in Transportation Systems, Pursula M, Niittymäki J (eds). Kluwer: Boston, 2001; 49-79.

9. García R, Marín A. Parking capacity and pricing in park’n ride trips: a continuous equilibrium network design problem. Annals of Operations Research 2002; 116:153-178.

10. Laporte G, Marín A, Mesa JA, Ortega FA. An integrated methodology for the rapid transit network design problem. Lecture Notes in Computer Science. Algorithmic Methods for Railway Optimization 2007; 4359:187-199.

11. Marín A. An extension to rapid transit network design problem. TOP 2007; 15:231-241.

12. Fortz B, Labbé M. Two-connected networks with rings of bounded cardinality. Computational Optimization and Applications 2004; 27:123-148.

13. Fortz B, Mahjoub AR, McCormick ST, Pesneau P. Two-edge connected subgraphs with bounded rings: polyhedral results and branch-and-cut. Mathematical Programming 2006; 105:85-111.

14. Grötschel M, Monma CL, Stoer M. Polyhedral and computational investigations for designing communication networks with high survivability requirements. Operations Research 1995; 43:1012-1024.

15. Lou Y, Yin Y, Lawphongpanich S. A robust approach to discrete network designs with demand uncertainty. Transportation Research Record: Journal of the Transportation Research Board 2009; 2090:86-94.

16. Yin Y, Lawphongpanich S. A robust approach to continuous network designs with demand uncertainty. In Transportation and Traffic Theory, Allsop RE, Bell MGH, Heydecker BG (eds). Elsevier: Amsterdam, 2007; 111-126.

17. Lo HK, Tung Y-K. Network with degradable links: capacity analysis and design. Transportation Research Part B 2003; 37:345-363.

18. Yang H, Bell MGH. Models and algorithms to road network design: a review and some new developments. Transport Reviews 1998; 18:257-278.

19. Rockafellar RT, Wets RJ-B. Scenarios and policy aggregation in optimization under uncertainty. Mathematics of Operation Research 1991; 16:119-147.

20. Malcolm S, Zenios SA. Robust optimization for power systems expansion under uncertainty. Journal of the Operational Research Society 1994; 45:1040-1049.

21. Mulvey JM, Vanderbei RJ, Zenios SA. Robust optimization for large-scale systems. Operations Research 1995; 43:264-281.

22. Nikulin Y. Robustness in Combinatorial Optimization and Scheduling Theory: An Annotated Bibliography, Manuskripte aus den Instituten für Betriebswirtschaftslehre der Universität Kiel, Publication 606, 2006.

23. Ben-Tal A, Nemirovski A. Robust convex optimization. Mathematics of Operations Research 1998; 23:769-805.

24. Ben-Tal A, Nemirovski A. Robust optimization - methodology and applications. Mathematical Programming 1999; 92:453-480. 
25. Bertsimas D, Sim M. Robust discrete optimization and network flows. Mathematical Programming 2003; 98:49-71.

26. Bertsimas D, Sim M. The price of robustness. Operations Research 2004; 52:35-52.

27. Immers LH, Yperman I, Stada JE, Bleukx A. Reliability and robustness of transportation networks. Problem survey and examples. Proceedings of the Nectar, Cluster 1 Meeting, Amsterdam, 2004.

28. Immers LH, Stada JE, Yperman I, Bleukx A. Robustness and resilience of transportation networks. Proceedings of the 9th International Scientific Conference MOBILITA, Bratislava, 2004.

29. Marcotte P, Patriksson M. Traffic equilibrium. In Handbooks in Operations Research and Management Science, Volume 14, Barnhart C, Laporte G (eds). North-Holland: Amsterdam, 2007; 623-713.

30. Marín A, Jaramillo P. Urban rapid transit network design: accelerated benders decomposition. To appear in Annals of Operations Research 2009; 169:35-53.

31. Marín A, García-Ródenas R. Location of infrastructure in urban railway networks. To appear in Computers, Operations Research 2009; 36:1461-1477.

32. Garzón A, Mesa JA, Ortega F. A Rapid Transit Model in Regard to Transfers, Advanced OR and AI Methods in Transportation. Publishing House of Poznan University of Technology: Poznan, 2005; 212-217.

33. Garzón A, Martínez FJ, Melián B, Mesa JA, Moreno JA, Ortega FA. Metaheurística GRASP para el Diseño de Redes de Tránsito Rápido. IV Spanish Conference on Metaheuristics, and Evolutionary Algorithms (Maeb, 2005). Thomson-Paraninfo, Granada, Spain, pp. 601-608, 2005.

34. Cascetta E. Transportation Systems Engineering: Theory and Methods. Kluwer: Boston, 2001. 